\title{
Research Online Mobile: Towards a Universal Collaboration Research Environment
}

\author{
Taotao Shi, Yongzheng Ma, Guangyuan Liu, Jianjun Xie, Hongwei Yang \\ Computer Network Information Center, Chinese Academy of Sciences, Beijing, China \\ Email: shitaotao@cnic.cn,myz@cnic.cn, lgy@cnic.cn, xiejj@cnic.cn, yhw@cnic.cn
}

Received 2013

\begin{abstract}
The collaboration research environment aims to support a team of researchers who are working coordinately over the Internet. With the popularity of mobile phones and the widespread use of the high-speed wireless network, people are demanding a universal collaboration research environment in which they can cooperate wherever they are. In this paper, we present the Research Online Mobile, a universal collaboration research environment based on the Duckling platform, which is an open source software. The framework, key services and applications of the Research Online Mobile are also elucidated. The Research Online Mobile has been released and used by several research communities.
\end{abstract}

Keywords: Research Online Smartphones Android iOS

\section{Introduction}

The Internet has profoundly changed and will continue to change our work style. A Software system that supports a team of researchers who are working coordinately over the Internet is called the collaboration Research Environment [1].

The collaboration research environment actually originates from the Computer Supported Cooperative Work (CSCW) [2]. On the basis of the CSCW technology, the collaboration research environment exploits various types of cyberinfrastructure, such as high-speed networks, high performance computing, and mass storages, to integrate and share resources. Thus, it can support a wide range of exchanges and cooperations, including collaborative edition, remote control, computer simulation, realtime communication and multimedia interactions, among a team of researchers from different disciplines, organizations, and institutions.

\subsection{The Duckling Platform}

Based on concepts and key technologies of the collaboration research environment, Computer Network Information Center, Chinese Academy of Sciences (CNIC-CAS) has developed the Duckling platform which provides visual displays and applications of various types of research infrastructure for researchers by Web 2.0. According to some special demands from researchers, a lot of plug-ins have been released based on the APIs provided by the Duckling. Currently, there are a spectrum of plug-ins covering conference services, universal commu- nications, organizations and document management plugins.

The Duckling platform provides a common research collaboration basement and forms a set of virtual research collaboration oriented software operating environments with application integration technologies. The Duckling platform is an Internet-based virtual collaboration infrastructure for scientific communities. With collaboration tools of the Duckling platform, researchers can create private virtual research spaces to exchange and communicate on research topics.

The latest version of Duckling is 3.0. Its source code and deployment package were officially released on the world's largest open-source site SourceForge on March 17, 2010.

\subsection{Research Online}

By means of the cloud service, Research Online (http://www.escience.cn) is based on the Duckling platform and provides transparent collaboration services for researchers, including collaborative edition, document storage, research management, scientific data processing, high performance computing, and data visualization. It integrates distributed cyberinfrastructure of Chinese Academy of Sciences and merges scientific data from various disciplines.

Currently, about 100 groups of researchers have their collaboration spaces in Research Online. They can have a unique name, distinctive user interface style, and customized home page through a series of steps. It can en- 
able a virtual research team carry out the collaborative researching work such as document sharing, data collaboration, instant messaging and project management. Since Research Online is responsible for the maintenance and management of every collaboration space, researchers only need to focus on their research work.

\subsection{Research Online Mobile}

On Research Online, users can create their own collaboration spaces in an easy way, which can support a team to disseminate their scientific research work and publish the relevant information. By December 31, 2011, there were already 314 team spaces, 456 personal spaces, 883 users on Research Online.

Research Online can ensure a smooth collaboration process in idea exchange, theoretical discussions and project implementations. However, there are more conferences, business trips and fragmented time nowadays, simple operations like following up projects, sharing knowledge and browsing information on Research Online can be completed without the desktop devices, which is becoming more and more popular. Moreover, with the popularity of mobile phones and the widespread use of the high-speed wireless network, people are demanding that they can access Research Online wherever they are. Research Online Mobile is to fulfill this requirement.

This paper presents Research Online Mobile which supports researchers' access their collaboration spaces in Research Online anywhere and anytime by smartphones. The framework, key services and applications of the Research Online Mobile are also clarified. Research Online Mobile has already been released and used by several research communities.

\section{Framework}

\subsection{Characteristics}

Research Online Mobile has the following features: 1)
Low-cost construction: no software or hardware special requirements in server-side deployment. 2) Transmitting quickly: fast access to the page, and provide a pleasant user experience. 3) Easy to navigate: everyone can browse viewpoints and documents anytime, anywhere. 4) Knowledge sharing in multimedia: multimedia is the main way in knowledge sharing. 5) Easy to extend: make it possible to maintain and extend new functions.

\subsection{Architecture Design}

1) OAuth Authentication: OAuth [3] is an open standard for authorization. It does not allow a third party to reach the user's account information. That is to say, a third party can apply for the authorization of the user's resources without sharing user's username and password, then the authorization between different applications is reliable and safe. The abstract flow illustrated in Figure 1 describes interactions between the user, Research Online Mobile, and the resource server.

In the first step, a user requests the protected resources from the resource server through Research Online Mobile. Second, Research Online Mobile requests an unauthorized request token from the resource server. Then, the server validates user's username and password, and if valid, it issues an unauthorized request token with corresponding encryption key (step 2 to step 4). Third, the resource server authenticates the unauthorized request token presented by Research Online Mobile, if valid, it returns the authorization grant, an authorized request token, and its encryption key (step 4 to step 5). Fourth, the server authenticates the client and validates the authorization grant, If valid, it would issue an access token (step 6 to step 7). Thus, with the access token validated by the server, Research Online Mobile can get the protected resources (step 8 to step 9). Finally, the user can access the protected resources in Research Online Mobile as shown in the step 10 .

2) System Architecture: As shown in Figure 2, Research

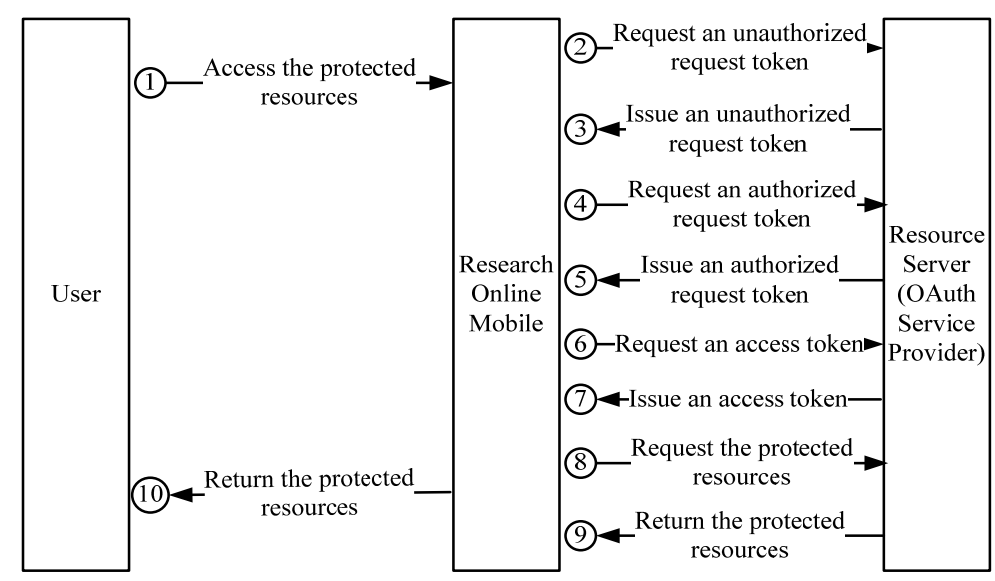

Figure 1. OAuth authentication process. 
Online Mobile can be deployed in the portable devices, such as smartphones, tablet PCs, and iPod touch.

During the login process, a user is identified by the OAuth Authentication. With the interface on the client, the user information and the service requests are submitted to the server. The server-side interface extracts the appropriate data from the background database and packages them into packets, then sends them to the client. When receiving responses, the client parses the corresponding data packets from the server and displays them to the user. Meanwhile, the server is responsible for saving data from the client to the database.

\subsection{System Design}

1) Server-side Design: In Research Online, any web page can be seen as a carrier of information and knowledge, just as an article. After writing ideas on a page in Research Online, or uploading any type of file as an attachment, users can share with their team members.

As shown in Figure 3, "Login Authentication" is responsible for OAuth authentication and authorization when users sign in. "Query” and "Search" are designed to get the appropriate data from the server-side's database, including the participation of users, collection of pages, and list of corresponding pages and page contents. Then, the server packages them into Json format and sends them to the client for parsing and displaying. "Access Page Content" is to obtain the page content from the server according to its URL, and then the page would be displayed in the client's Web View control. "Check Version for Update" supports version comparison and upgrade. "Saving Media Data To Database" can save pictures and recording files coming from the client to the server-side's database.

2) Client Design: The interface on the client uses a relative layout, which ensures that the client is adaptive to different resolutions of the screen. The list of pages displays the title, creator, and creation date of the page. User can access the appropriate content in the Web View by clicking on an item in the list to get the page's URL. On Research Online Mobile, users can visit the team's information, browse documents, share the knowledge in multimedia, and perform other operations anytime, anyplace even if when they are offline. When the client gets response from the server, it would make a data copy to its local database for offline using. "Navigation” aims to help users focus on the pages which have been modified or created recently. "Collection" is similar to the folder, through which users can get the results of the preliminary classification of documents and materials. "Team Updating" shows the pages updated recently sorted by date. "More" includes the system services, such as choosing

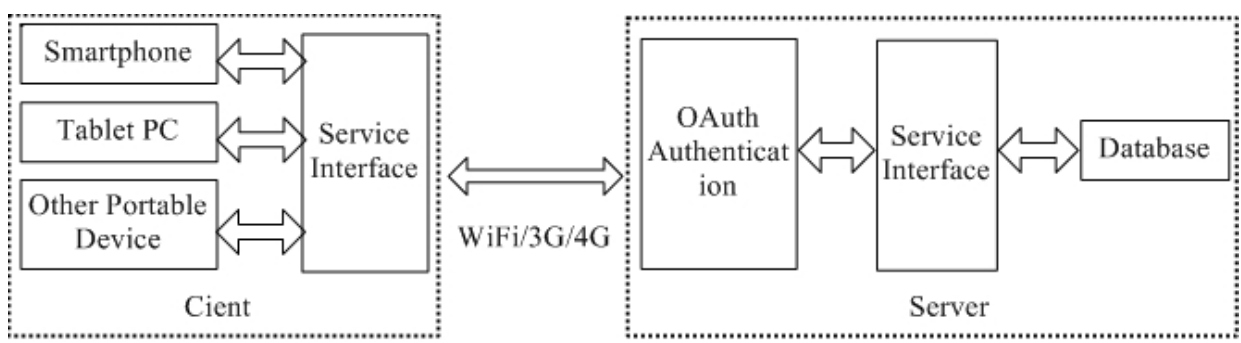

Fiure 2. Research online mobile architecture.

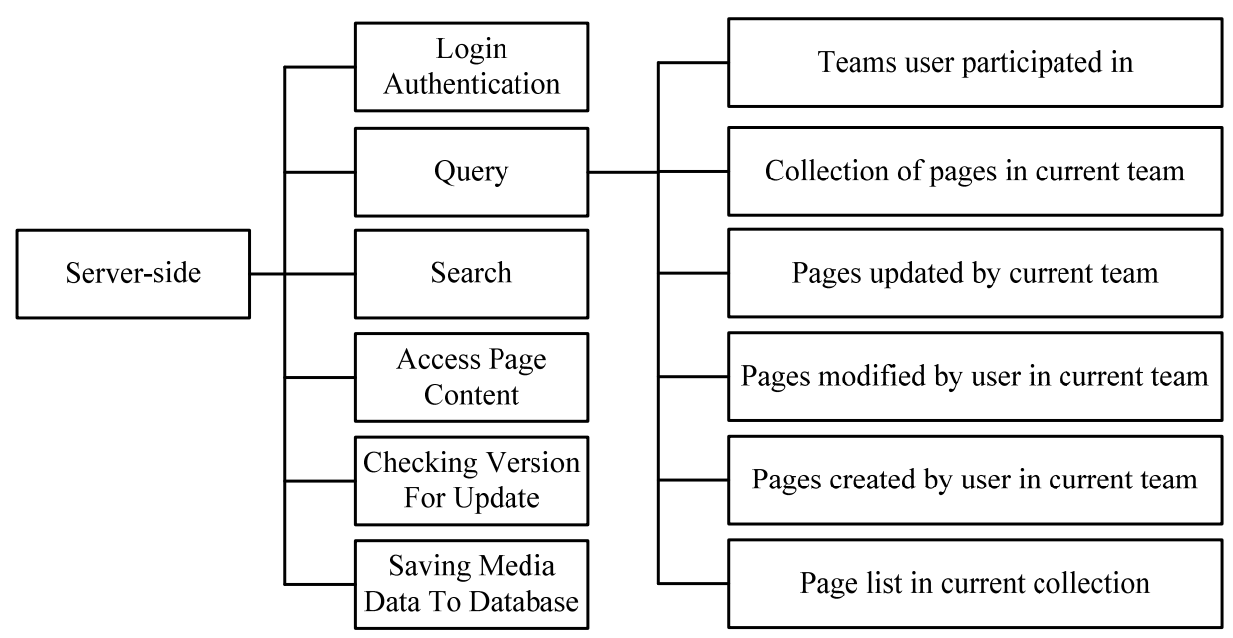

Figure 3. Server-side design. 
teams, re-login, canceling account, clearing cache, checking for updates and switching servers. "Viewed Recently" can help users find the pages concerned more from the local database. It also provides an efficient search engine to help users quickly find the desired documents in "Search". Additionally, in order to create a simple, fast user experience, and reduce the burden of entering text, the multimedia is taken as the main way of inputting, detailed in "Inputting". Client design is shown in Figure 4.

3) Special Processing: Smartphones are becoming the mainstream. They have very large coverage on the mobile phone market and numerous users. However, due to the growing network traffic produced by more and more applications, the wireless network speed is still the key factor. In order to provide users a pleasant user experience, there are several special technologies in transmission and navigation in Research Online Mobile:

(1) GNUzip (GZIP) [4] is a GNU free software tool which is designed for compression. When the client requests several pages, the server compresses them via GZIP. Generally, plain text content is compressed to $40 \%$ of its original size, which allows users to have a faster transmission speed. (2) Due to the size and its resolution, the screen of the portable device can only accommodate a relatively small amount of information. To allow users to focus on their research topics, the pages returned from the server have no banners, borders, or decorations, but only key information, including objectives, planning, scheduling and knowledge perspectives, which can fit in the screen of the portal device. (3) Research Online intends to build a knowledge-based system like the Wiki, facilitating users to browse pages by embedding links in the pages. However, in order to keep users from browsing outside the current application, which costs more time and produces unnecessary network traffic, Research Online Mobile cancels the hyper- links in the pages.

\section{Implementation}

\subsection{Smartphone Platform}

The 2012 Mobile Future Focus Report released by ComScore shows that nearly 42 percent of U.S. mobile phone users, 44 percent of the five European countries' mobile phone users now owns a smartphone. Another key conclusion is that the competition between different smartphone platforms is fiercer. Currently, the Android platform accounted for nearly half of the market, and iOS nearly 30\%, which means that Android and iOS are prevailing in most markets [5].

With the rapid progress of the next generation wireless communication, it is foreseeable that the smartphone is essential for everyone in obtaining new information. Since we usher in the era of the knowledge economy, there are more meetings/conferences, business trips and fragmented time nowadays. Therefore, Research Online Mobile is a great improvement to Research Online with the mobility, convenience to use, and the advantage of the wireless network.

\subsection{Android-Based}

1) Android Architecture Analysis: Launched by Google in 2007, Android is an operating system designed for mobile devices. It's the first one to create a truly open mobile development platform for the portable device. Android's core system services rely on the Linux 2.6 kernel which includes some $\mathrm{C} / \mathrm{C}++$ library to provide services for developers through the Android application framework. Android Runtime includes a core library and the Dalvik virtual machine which is developed by Google in order to save resources on the phone. The Android Software Development Kit (SDK) provides all the

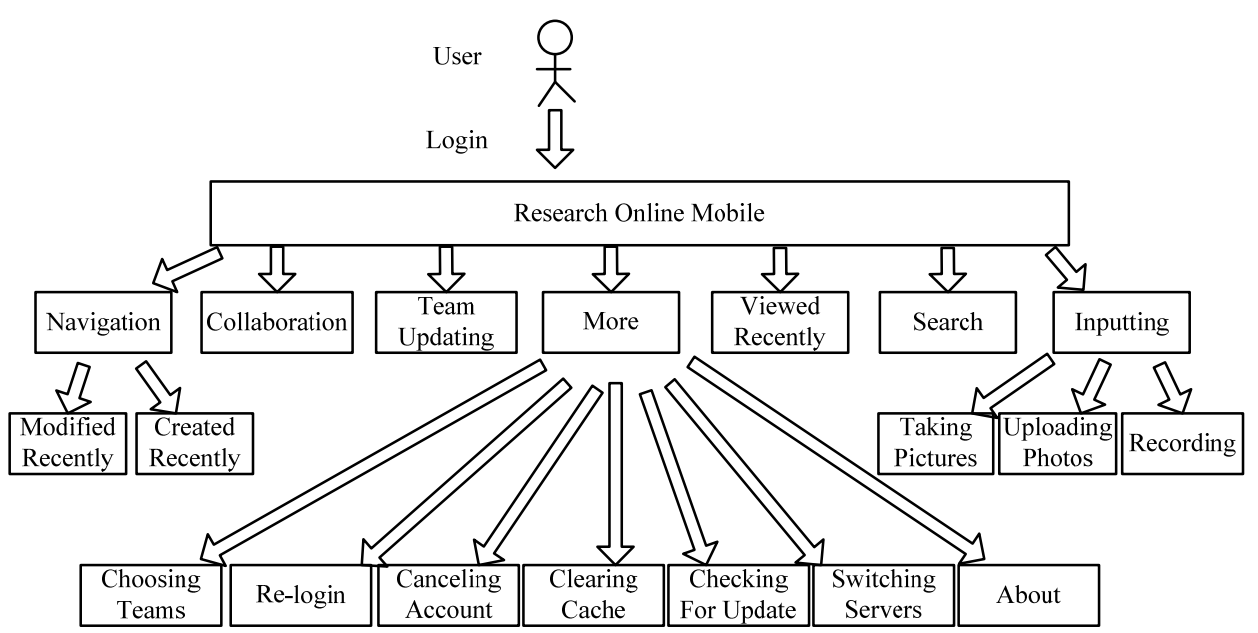

Figure 4. Client design. 
tools and APIs for development [6]. Android platform supports Java programming language for application development. Programmers can use the Eclipse integrated development environment to install the ADT tool for the mobile development.

We choose the Android as one of our target mobile device platforms because of its openness and wide industry support. The source code of the Android is publicly available and currently 50 leading telecom, hardware, software companies are registered as its members [7]. Thus, our challenge is to implement the application on the platform to facilitate the exchange for researchers.

2) Detailed Implementation: As shown in Figure 5(a), users should login with Duckling pass. "Inputting" in the bottom of the Tab Bar supports users to record the knowledge and share with other team members by taking pictures, uploading photos and recording. Pictures from the smartphones' camera or the system album should be converted to the Bitmap format then to a byte stream, finally uploaded to the server or saved locally. If the photo is not uploaded successfully but saved, the system must take the initiative to refresh the media library and upload it again. As shown in Figure 5(b), "Recording" is implemented with MediaRecorder. "Recording" needs to determine the saving file path in advance to ensure a smooth recording process, which is different from taking pictures. As recording is complete, users can choose to playback, upload or delete. On Android, the list of pages uses List View to display the page information. As shown in Figure 5(c), users can get the corresponding page content after selecting one item in the list. The Android-based Research Online Mobile supports version updating. When a new version is available, users can upgrade with the latest apk file downloaded from serverside (http://www.escience.cn/apks/maone-latest.apk) and installed automatically.

\section{3. iOS-Based}

1) iOS Architecture Analysis: iOS is Apple Inc.'s mobile operating system. iOS's system consists of four levels: the Core OS layer, the Core Services layer, the Media layer and the Cocoa Touch layer. System operation occupies about $240 \mathrm{MB}$ of memory space [8].

The Cocoa Touch layer contains the required framework to create iOS applications. The Media layer contains graphics technology, audio and video technologies which combined with each other to bring the best multimedia experience for mobile devices. The Core Services layer provides basic system services for all applications. The Core OS layer is the basis of a lot of other technologies [9]. iOS platform software development uses Objective-C language on the MAC computer, with Apple Xcode (the underlying platform of development) and Interface Builder (responsible for visual and graphic design of MAC) as the uniform development tools.

2) Detailed Implementation: "Team Updating" is the home page when entering Research Online Mobile. It shows that any additions or modifications of the page content in a team. Table View on its interface layout can be dropped down to refresh, which is implemented with an open-source framework named EGORefreshTableHeaderView [10]. It can bring users a pleasant experience as shown in Figure 6(a). The attribute sourceType of UIImagePickerController in iOS can tell whether the picture is from system camera or the media library. Users can get a local copy automatically once uploading pictures successfully, shown in Figure 6(b). The application implements recording with AVAudioRecorder and playback with AVAudioPlayer, and then users can choose to upload or delete local recording files in UIActionSheet. As shown in Figure 6(c), "More" makes it possible for users to switch servers, choose participating teams, cancel the account and clear the cache.

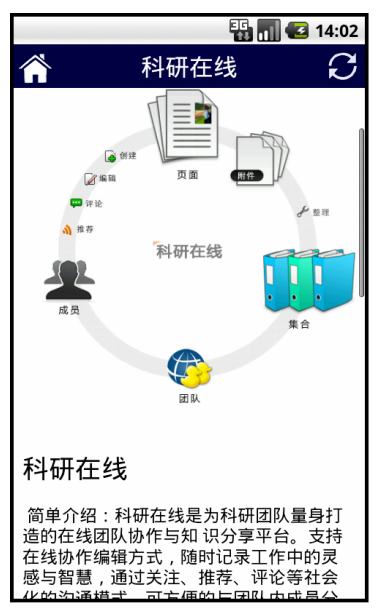

(a)

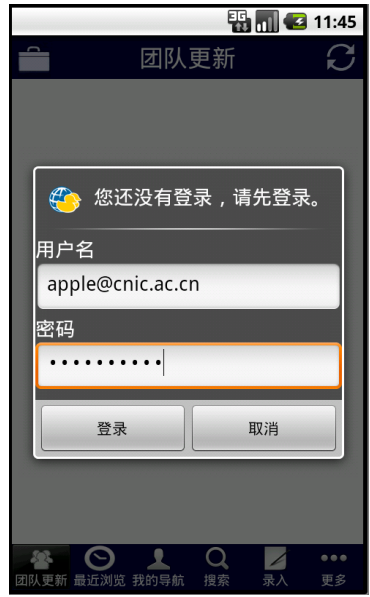

(b)

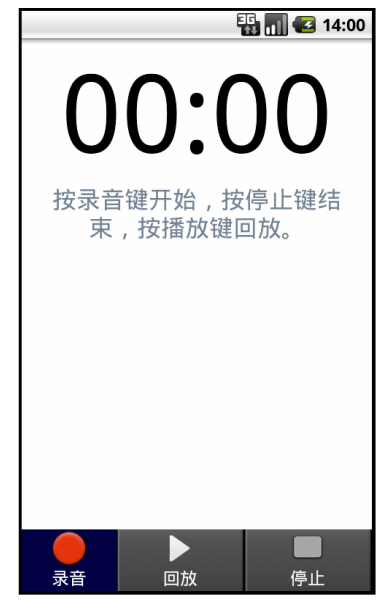

(c)

Figure 5. Android-based implementation; (a) Login; (b) Recording; (c) Page content. 


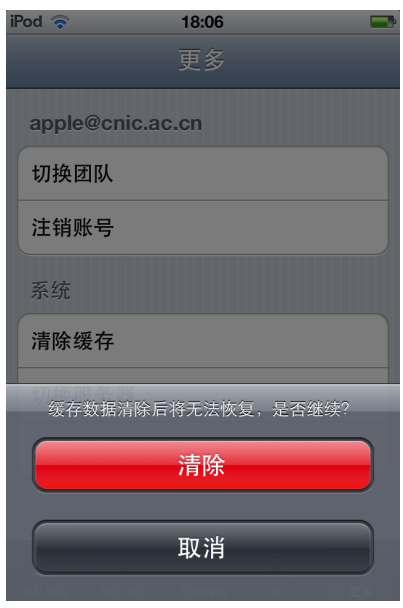

(a)

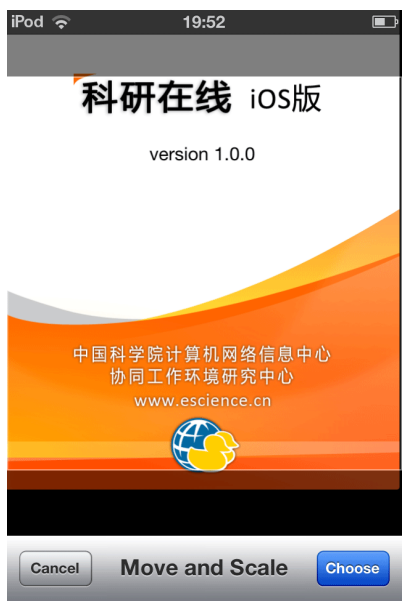

(b)

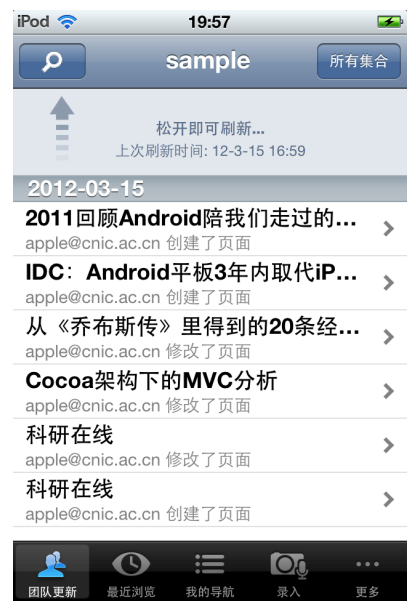

(c)

Figure 6. iOS-based implementation. (a) Team updating; (b) Photo uploading; (c) Clearing cache.

\section{Conclusions}

Research Online provides transparent Internet-based collaboration services for researchers with Duckling. It can integrate and share distributed and heterogeneous resources in the application of scientific research. This paper presents the Research Online Mobile framework, Android-based and iOS-based implementation. It can achieve researchers' urgent demands to access their collaboration spaces in Research Online anywhere and anytime by smart phones.

Research Online Mobile has been released and used by several research communities. Furthermore, with the rapid development of mobile computing technology and the great needs for Research Online, Research Online Mobile will certainly play an increasingly important role. Thus, more comprehensive application modes should be studied and improved in the future work.

\section{Acknowledgements}

This research is supported in part by the Chinese Academy of Sciences-Science grant INFO-115-D01 and the CNIC Innovation Foundation under Grant CNIC_CX_ 10004.

\section{REFERENCES}

[1] N. Kai, et al. "Special Issue of Scientific Collaboration and Its Typical Research Work," Current Work of Informatization, Vol. 20, 2010, pp. 14-30.

[2] G. L. Shi, Y. Xiang and G. X. Yang, "Theory and Practice of Computer Supported Cooperative Work," Publishing House of Electronics Industry, Beijing, 2000.

[3] The OAuth 2.0 Authorization Protocol draft-ietf-oauthv2-20, 2011.

[4] The Gzip Home Page. http://www.gzip.org

[5] http://www.199it.com/archives/25088.html

[6] R. Meier, "PROFESSIONAL Android"TM2 Application Development,” Wiley Publishing, Inc., Indianapolis, Indiana, 2010.

[7] Open Handset Alliance. http://www.openhandsetalliance.com/ oha_members.html

[8] H. Karen, "Macworld Expo: Optimised OS X Sits on 'Versatile' Flash,” Macworld, 2007.

[9] Developer, “iOS Technology Overview,” Apple Inc., 2011.

[10] http://www.cocoanetics.com/2009/12/how-to-make-a-pull -to-reload-tableview-just-like-tweetie-2/ 